\title{
In vitro study on shRNA-mediated reduction of testis developmental related gene 1 expression and its effects on the proliferation, invasion and apoptosis of NTERA-2 cells
}

\author{
YU GAN, JIANFU YANG, YONG WANG, ZHENGYU TAN, XIANZHEN JIANG and YUXIN TANG
}

Department of Urology, The Third Xiangya Hospital of Central South University, Changsha, Hunan 410013, P.R. China

Received July 25, 2014; Accepted March 16, 2015

DOI: $10.3892 / \mathrm{ol} .2015 .3219$

\begin{abstract}
Testis developmental related gene 1 (TDRG1) is a novel human testis-specific gene. TDRGl is differentially expressed in cancerous tissue compared with normal testicular tissue and demonstrates a unique expression pattern in normal testes; therefore, this gene may be involved in the occurrence and development of testicular germ cell tumors (TGCT). In the present study, the expression level of TDRG1 was downregulated in human TGCT NTERA-2 cells by RNA interference (RNAi) in order to investigate the association between TDRG1 and TGCT. The TDRG1 mRNA and protein expression levels in NTERA-2 cells were significantly inhibited following transfection with specific RNAi plasmids. The ability to proliferate (inhibited by $15.4 \%$ at day 3 and $26.1 \%$ at day $5 ; \mathrm{P}<0.001$ ) and invade (reduced by $49.1 \% ; \mathrm{P}=0.01$ ) in vitro was suppressed in cells in which the expression level of TDRG1 was reduced, and a corresponding increase in the apoptotic potential was observed (the early apoptotic potential and total apoptotic potential were increased by $75 \% ; \mathrm{P}=0.019$ and $54.8 \% ; \mathrm{P}=0.009$, respectively). The results of the present study indicated that the biological behavior of NTERA-2 cells is associated with TDRG1 expression levels, and that this gene may be a novel target candidate in the treatment of TGCT.
\end{abstract}

\section{Introduction}

Testicular germ cell tumors (TGCTs) are a type of solid tumor that are most commonly observed in young men aged 15-35 years (1). There is a relatively large difference in the incidence of TGCT among different regions or different races, but the worldwide incidence of TGCT has increased in the last $30-40$ years $(2,3)$. However, $>95 \%$ patients diagnosed at an early stage are cured (4). TGCT treatment is associated

Correspondence to: Professor Yuxin Tang, Department of Urology, The Third Xiangya Hospital of Central South University, 138 Tongzipo Road, Changsha, Hunan 410013, P.R. China E-mail: rainson27@126.com

Key words: TDRG1, NTERA-2, siRNA, cell biological behavior, in vitro with chronic side effects; patients with advanced-stage cancer are less sensitive to comprehensive treatments, resulting in a poor prognosis (5). The molecular biology of TGCT remains largely unknown, but the onset and development of the disease is a process that involves multiple genes (6). Increased understanding of the molecular biology of TGCT is essential in order to define targeted treatments and identify novel markers for diagnosis and prognosis.

Testis developmental related gene 1 (TDRG1; GenBank ID, DQ168992) is specifically expressed in human testicular spermatogenic cells, but not in non-reproductive tissues. Furthermore, expression levels of TDRG1 are relatively increased in young men aged 15-33 years (7). Notably, all TGCT originate from the spermatogenic cells and the high-risk ages for the development of TGCT are consistent with the expression pattern of TDRG1. Previous studies have demonstrated the abnormal expression of TDRG1 protein in TGCT compared with non-malignant human testicular tissues (8). Therefore, it is possible that TDRG1 may be associated with the onset and development of TGCT.

The use of short hairpin (sh)RNA to silence a target gene has become a conventional and accurate method (9). In the present study, RNA interference (RNAi) was used to downregulate the expression level of TDRG1 in TGCT NTERA-2 cells. The effects of reduced TDRG1 expression levels on NTERA-2 cells were observed. The biological behavior of NTERA-2 cells with reduced TDRG1 expression was detected and compared with control groups and the association between these biological behaviors and TDRG1 was analyzed.

\section{Materials and methods}

Cell culture. The human TGCT NTERA-2 cell line was obtained from the Institute of Biochemistry and Cell Biology, Chinese Academy of Sciences (Shanghai, China) and cultured in Dulbecco's modified Eagle's medium (DMEM; Gibco Life Technologies, Carlsbad, CA, USA) supplemented with $10 \%$ fetal bovine serum (FBS; Invitrogen Life Technologies, Carlsbad, CA, USA) at $37^{\circ} \mathrm{C}$ in a humidified atmosphere of $5 \% \mathrm{CO}_{2}$.

TDRG1-targeting siRNA expression vector. Two previously constructed recombinant plasmid vectors were used in the present study (10). The recombinant plasmids were named 
pGPU6/GFP/Neo-shRNA486 (psh486) and pGPU6/GFP/Neo-shRNA-control (pshneg), respectively. The DNA concentration and purity of the plasmids was detected by ultraviolet spectrophotometry (SmartSpec 3000; Bio-Rad Laboratories, Inc., Hercules, CA, USA) then they were stored at $-20^{\circ} \mathrm{C}$. The shRNA486 sequence (the mRNA coding region of 486-506 is in upper case): Sense 5'-caccGCGCAGGATCAAGC TACAATGttcaagagaCATTGTAGCTTGATCCTGCGCtttttt g-3' and antisense 5'-gatccaaaaaaGCGCAGGATCAAGCTA CAATGtetcttgaaCATTGTAGCTTGATCCTGCGC-3'. The negative control sequence (the target sequence that has no homology with the human gene is in upper case): Sense, 5'-caccGTTCTCCGAACGTGTCACGTCcaagagaTTACGTGA CACGTTCGGAGAAttttttg-3' and antisense 5'-gatccaaaa aaTTCTCCGAACGTGTCACGTAAtctctTGACGTGACAC GTTCGGAGAAc-3'.

Transfection and grouping. NTERA-2 cells were passaged with $0.25 \mathrm{~g} / 1$ trypsin (GE Healthcare Life Sciences, Logan, UT, USA) and seeded into 6 -well plates at a density of $4.5 \times 10^{4}$ cells/well. When the cells reached $\geq 70 \%$ confluence, they were transfected in 3 groups as follows: i) Control group (no transfection); ii) negative control group (pshneg transfection); and iii) shRNA486 group (psh486 transfection).

Following the Lipofectamine 2000 (Invitrogen Life Technologies, Carlsbad, CA, USA) manufacturer's instructions, the plasmids described above and the liposome were diluted separately with Opti-MEM (Invitrogen Life Technologies). The effective ratio of plasmid:liposome was 1:2.5 $(\mu \mathrm{g}: \mu \mathrm{l})$. The diluted plasmid and liposome were mixed and incubated at room temperature for $20 \mathrm{~min}$ to allow complex formation. The transfection mixture was then added to each well with $2 \mathrm{ml}$ of FBS-free DMEM. Following $6 \mathrm{~h}$ incubation at $37^{\circ} \mathrm{C}$, the mixture was replaced with complete medium. Fluorescence microscopy (Nikon E800; Nikon Corporation, Tokyo, Japan) was used to confirm successful transfection after $48 \mathrm{~h}$.

RNA extraction and fluorescence quantitative polymerase chain reaction $(q P C R)$. The specific primers designed to amplify TDRG1 and GAPDH were designed and synthesized by Sangon Biotech Co., Ltd. (Shanghai, China). The following primer sequences were used: TDRG1, sense 5'-GAAGAGGAG GGAGGCAGTCT-3' and antisense 5'-GCCCAATTCCTC TTGACTGA-3'; GAPDH, sense 5'-ACCACAGTCCATGCC ATCAC-3' and antisense 5'- TCCACCACCCTGTTGCTG TA-3'. Total RNA was extracted from NTERA-2 cells using TRIzol reagent (Invitrogen Life Technologies). First strand cDNA synthesis was performed with the RevertAid H Minus First Strand cDNA Synthesis kit (Thermo Fisher Scientific, Inc., Waltham, MA, USA ). Fluorescence qPCR was performed in a total volume of $25 \mu \mathrm{l}$ containing $1 \mu \mathrm{l} \mathrm{cDNA}, 100 \mathrm{nM}$ of each TDRG1 or GAPDH primer, $12 \mu 1$ 2X SYBR Green PCR Master mix (Thermo Fisher Scientific, Inc.) and $\mathrm{ddH}_{2} \mathrm{O}$ to a total volume of $25 \mu \mathrm{l}$. All the procedures were performed according the manufacturer's instructions. The reaction parameters were $95^{\circ} \mathrm{C}$ for $5 \mathrm{~min}$ (pre-denaturation), followed by $20 \mathrm{sec}$ each at $94^{\circ} \mathrm{C}$ (denaturation), $56^{\circ} \mathrm{C}$ (annealing) and $72^{\circ} \mathrm{C}$ (elongation) for 40 cycles, then a final elongation step for $5 \mathrm{~min}$ at $72^{\circ} \mathrm{C}$. Preliminary experiments verified that the amplification efficiency of the target gene was similar to that of the reference gene GAPDH. The $2^{-\Delta \Delta C t}$ method was used for relative quantification and statistical analysis (11). Each sample was set up as three duplications and tested in triplicate.

Immunofluorescence. The cells cultured on glass coverslips were transfected according to the groups outlined previously. At 1, 3 or 5 days post-transfection, the cells were washed twice with phosphate-buffered saline (PBS) and fixed with ice cold 4\% paraformaldehyde (Sigma-Aldrich, St. Louis, MO, USA) for $20 \mathrm{~min}$ at room temperature, air-dried, and washed with PBS 3 times. The cells were then exposed to $0.25 \%$ Triton X-100 (Sigma-Aldrich) for $20 \mathrm{~min}$. Normal goat serum blocking fluid (10\%; Beijing Solarbio Science \& Technology Co., Ltd., Beijing, China) was applied to the fixed cells for $20 \mathrm{~min}$ at room temperature and then the blocking fluid was removed. Mouse anti-human TDRG1 monoclonal antibody (12) (1:1,000; ProMab Biotechnologies, Inc., Richmond, CA, USA) was applied to the cells and incubated overnight at $4^{\circ} \mathrm{C}$ (12). The cells were then washed with PBS 3 times, the secondary antibody Cy3-conjugated goat anti-mouse $\mathrm{IgG}$ (1:200; cat. no. A0521; Beyotime Institute of Biotechnology, Shanghai, China) was applied to the cells and the cells were incubated in the dark at $37^{\circ} \mathrm{C}$ for $40 \mathrm{~min}$. The cells were then washed with PBS 3 times, and stained with DAPI $(100 \mathrm{ng} / \mathrm{ml}$; Beijing Solarbio Science \& Technology Co., Ltd.), and the cells were again rinsed with PBS 3 times. The cells were observed using a fluorescence microscope (Nikon E800) and the integrated optical density (IOD) of the TDRG1 protein expression in 10 randomly selected visual fields was measured using 'Motic Fluo' software, version 1.0 (Motic China Group Corporation, Ltd., Shenzhen, China).

Cell proliferation assay. The effect of TDRG1 on the proliferation of NTERA-2 cells was measured using MTT (Sigma-Aldrich) assay. The transfected cells were cultured in 96-well plates at a density of $\sim 1 \times 10^{4}$ cells/well in a volume of $100 \mu \mathrm{l}$. At time points of 1,3 or 5 days, $20 \mu \mathrm{l} 5 \mathrm{mg} / \mathrm{ml}$ MTT solution was added to each well and the cells were incubated for $4 \mathrm{~h}$ at $37^{\circ} \mathrm{C}$. The supernatant in each well was aspirated and discarded and $150 \mu 1$ dimethyl sulfoxide (Amresco, LLC, Solon, OH, USA) was added to dissolve the formazan. A microplate reader (WD-2102A; Beijing Liuyi Instrument Factory, Beijing, China) was used to detect the OD value for each well at a wavelength of $570 \mathrm{~nm}$. Each sample was tested in triplicate.

Cell invasion assay. The in vitro invasion capability of the cells was measured using the Transwell invasion assay. The transfected cells were seeded at a density of $\sim 1 \times 10^{5}$ cells/well in the top chamber with a membrane (6-well insert, $8-\mu \mathrm{m}$ pore size; Corning Incorporated, New York, NY, USA) coated in Matrigel ( $1 \mathrm{mg} / \mathrm{ml}$; BD Biosciences, Franklin Lakes, NJ, USA). The cells were cultured in serum-free medium, and medium containing $10 \%$ FBS was used as a chemoattractant in the lower chamber. Following 24-h incubation, the chamber was removed, the Matrigel and the cells in the upper chamber were wiped with a cotton swab and discarded. The cells in the lower chamber were then fixed with 95\% alcohol for 15-20 min and stained with $0.5 \%$ eosin (Beijing Solarbio Science \& Technology Co., Ltd.) for $10 \mathrm{~min}$. The cells in 5 randomly selected 
A

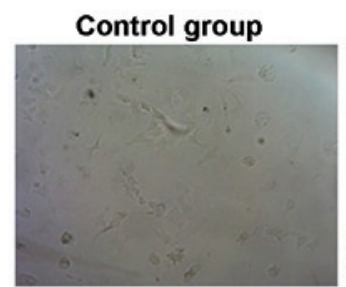

Negative control group

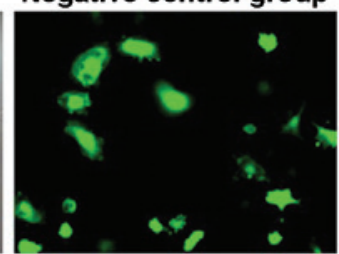

shRNA486 group

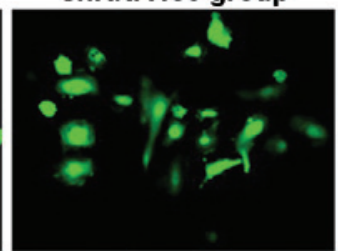

B

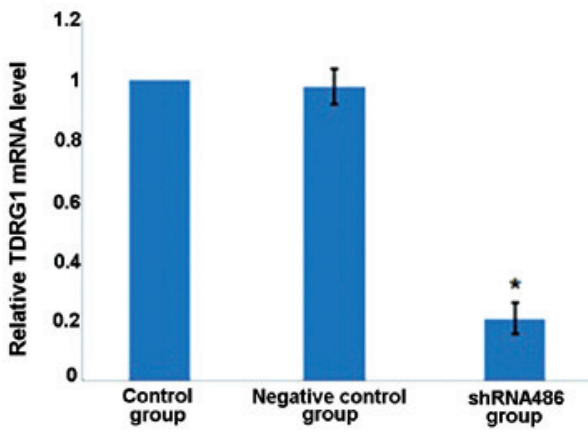

Figure 1. (A) The NTERA-2 cells in the control group did not demonstrate green fluorescence under the exciting wavelength. The transfection efficiency was $\geq 80 \%$ for the other two transfection groups. (B) Comparison of the relative TDRG1 mRNA expression levels between the three groups at 3 days post-transfection. The mRNA expression level of the TDRG1 gene in NTERA-2 cells transfected with psh 486 was reduced by 79\% ("P<0.05 vs. the control group). TDGR1, testis developmental related gene 1; shRNA, short hairpin RNA.

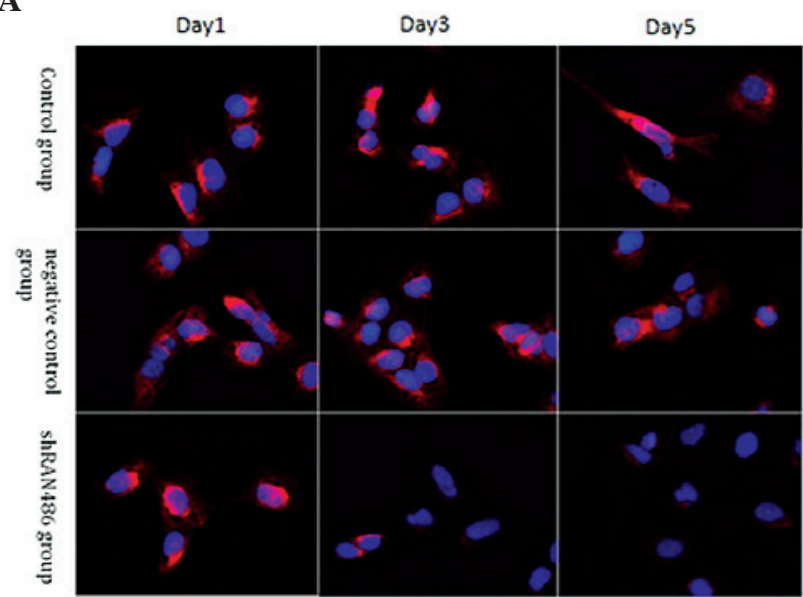

B

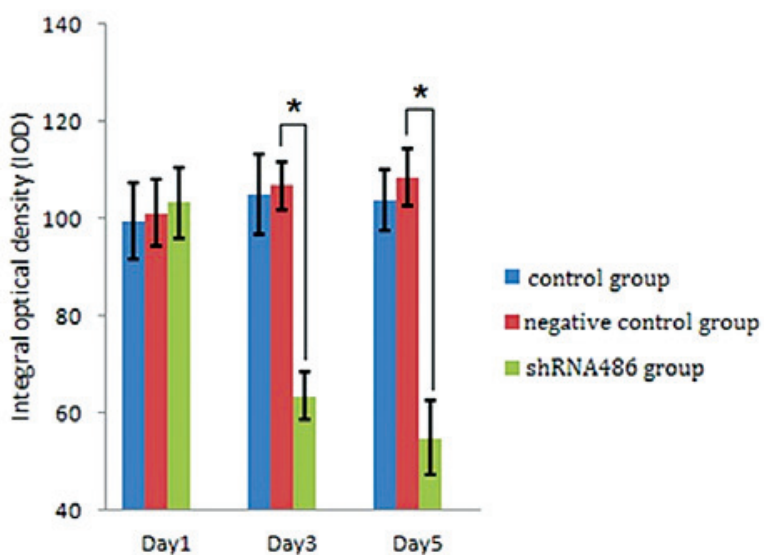

Figure 2. Immunofluorescence and the IOD value of the TDRG1 protein in the three groups. (A) The TDRG1 protein appears as red-orange fluorescence while the cell nucleus appears as blue. At days 3 and 5, the fluorescent signal of the TDRG1 protein in the shRNA486 group was reduced compared with day 1. (B) At days 3 and 5 post-transfection, the IOD value of the TDRG1 protein in the shRNA486 group was significantly reduced compared with the two control groups $($ ( $\mathrm{P}<0.05)$. This result indicates that the expression of the TDRG1 protein in NTERA-2 cells was reduced following effective transfection. IOD, integral optical density; TDGR1, testis developmental related gene 1; shRNA, short hairpin RNA.

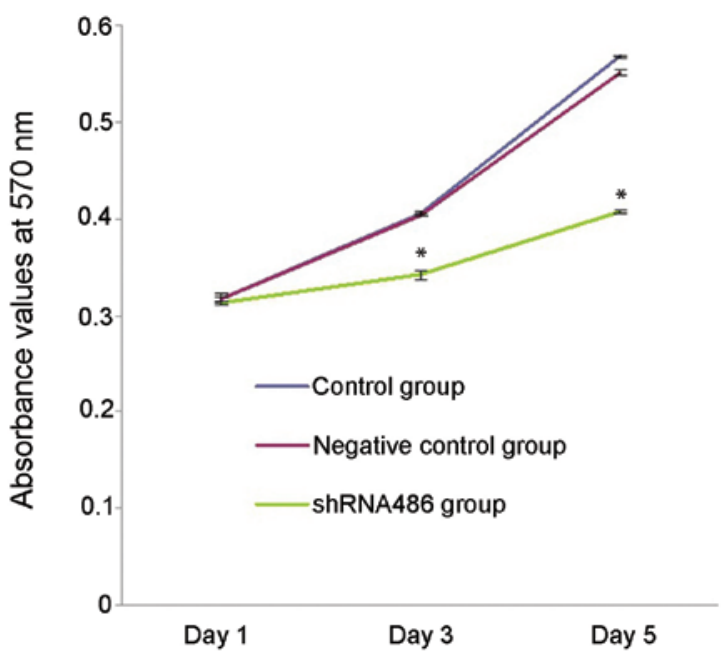

Figure 3. Detection of cell proliferation with MTT assay. A microplate reader was used to detect the optical density of each well at a wavelength of $570 \mathrm{~nm}$ at days 1,3 or 5 post-transfection. The average value was calculated for each time point and group. The average absorbance values of shRNA486 group at days 3 and 5 were significantly lower than the other two groups ( $\mathrm{P}<0.001)$ indicating that the in vitro proliferation ability of this group was inhibited. shRNA, short hairpin RNA. visual fields were counted under a light microscope (Olympus IX70; Olympus Corporation, Osaka, Japan), and the average value was recorded. This experiment was repeated 3 times.

Flow cytometric analysis. An annexin V-fluorescein isothiocyanate (FITC) and propidium iodide (PI) double-staining cell apoptosis test kit (KeyGene Inc., Nanjing, China) was used to detect the rate of apoptosis in each group. The cells were collected at $48 \mathrm{~h}$ following transfection and resuspended in the binding buffer containing annexin V-FITC and PI according to the manufacturer's instructions. All the samples were then analyzed using a FACScan flow cytometer (PT001374; BD Biosciences, San Jose, CA, USA) and the cells were classified as living cells, early apoptotic cells, advanced apoptotic cells and necrotic cells. The results were analyzed using BD FACSDiva software, version 6.1.3 (BD Biosciences) and the experiment was performed 3 times.

Statistical analysis. The results are expressed as the mean \pm standard deviation. SPSS software, version 13.0 (SPSS, Inc., Chicago, IL, USA) was used to perform statistical analyses 

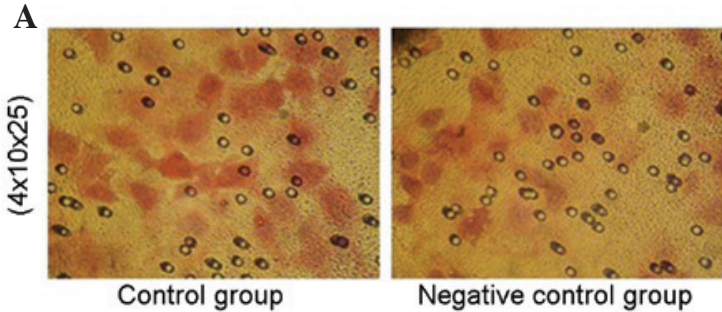

Negative control group

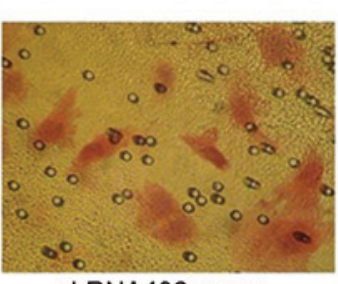

ShRNA486 group

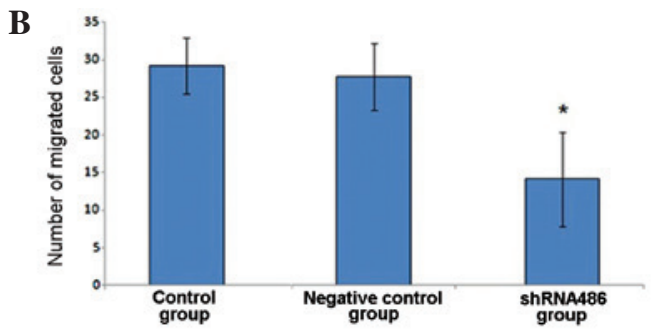

Figure 4. Analysis of cell invasion ability using a Transwell assay. (A) The cells penetrating the filter membrane were stained with eosin and appeared red under a light microscope. (B) Fewer cells penetrated the filter membrane in the shRNA486 group compared with the other two groups ("P<0.05). The in vitro invasion ability of the NTERA-2 cells transfected with the shRNA486 recombinant vector was inhibited by $\leq 49.1 \%$. shRNA, short hairpin RNA.
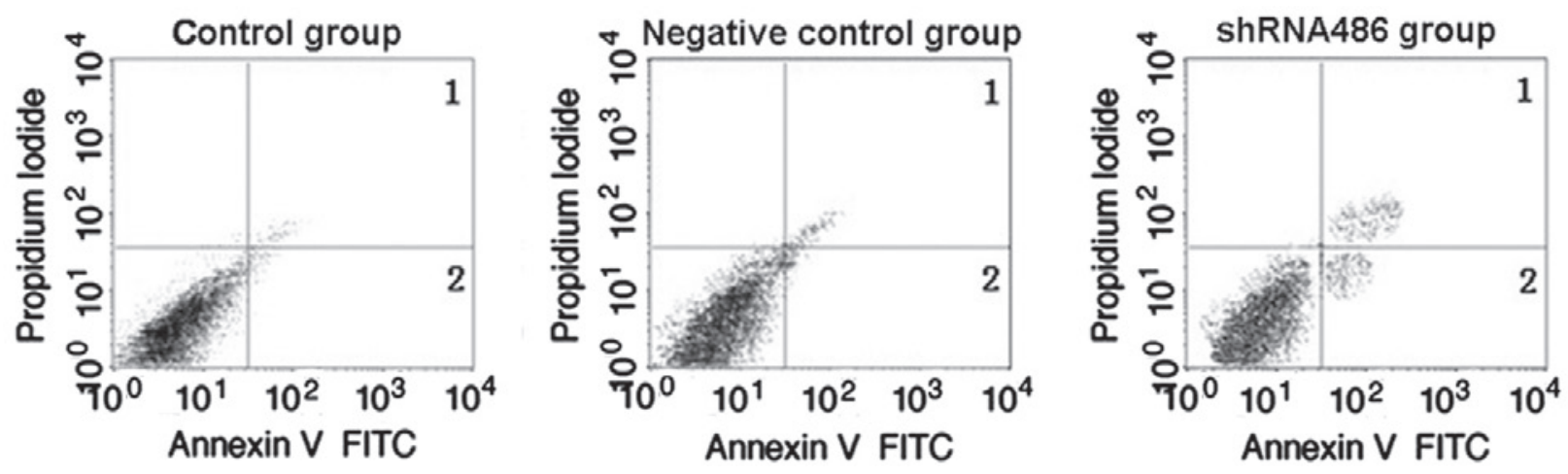

Figure 5. Measurement of cell apoptosis with annexin V/propidium iodide by flow cytometry. Quadrant 2 represents the proportion of early apoptotic cells, and quadrant 1 represents non-viable apoptotic cells and necrotic cells. The results indicated that silencing TDRG1 gene can induce apoptosis in NTERA-2 cells. TDRG1, testis developmental related gene 1; shRNA, short hairpin RNA; FITC, fluorescein isothiocyanate.

and the data were analyzed by analysis of variance. $\mathrm{P} \leq 0.05$ was considered to indicate a statistically significant difference.

\section{Results}

TDRG1 expression levels were downregulated following transfection. At 3 days post-transfection, the expression levels of GFP were observed using the fluorescence microscope (Fig. 1A). Two types of recombinant vector were successfully transfected into NTERA-2 cells. The relative level of TDRG1 mRNA expression in the negative control group was $0.99 \pm 0.04$ ( $\mathrm{P}>0.05$ vs. the control group) on day 3 , which was greater than that of the shRNA486 group $(0.21 \pm 0.03, \mathrm{P}<0.05$ vs. the control group; Fig. 1B). Immunofluorescence detection was used to observe TDRG1 protein expression following shRNA transfection (Fig. 2A). At 1 day post-transfection, the IOD values of the TDRG1 protein did not significantly differ among the three groups (Fig. 2B; P>0.05): Control group, 99.41 \pm 7.76 ; negative control group, 101.19 \pm 8.18 ; and shRNA486 group, 103.31 \pm 6.17 . However, at day 3 and 5 subsequent to transfection, the IOD value of the TDRG1 protein in the shRNA486 group was significantly lower compared with the other two groups (Fig. $2 \mathrm{~B} ; \mathrm{P}<0.05$ ). At 3 days post-transfection: Control group, 104.93 \pm 6.83 ; negative control group, 106.85 \pm 5.00 ; and shRNA486 group, 63.46 \pm 6.00 . At 5 days post-transfection: Control group, 103.87 \pm 7.32 ; negative control group, 108.52 \pm 4.89 ; and shRNA486 group, 54.92 \pm 7.59 . Notably, the IOD value of the TDRG1 protein in the negative control group was not significantly different compared with the control group $(\mathrm{P}>0.05)$.
TDRG1 silencing reduces the proliferation ability of NTERA-2 cells. At 1, 3 and 5 days post-transfection, the in vitro proliferation ability of NTERA-2 cells in each group was assessed using MTT assay (Fig. 3). The proliferation ability of NTERA-2 cells transfected with the psh486 was significantly reduced compared with the cells in the control group on day $3(15.4 \%$ reduction, $\mathrm{P}<0.001)$ and day $5(26.1 \%$ reduction, $\mathrm{P}<0.001)$ post-transfection. No significant differences in proliferation capacity were observed at days 1,3 or 5 $(\mathrm{P}>0.05)$ between the control and negative control group.

TDRG1 silencing reduced the invasion ability of NTERA-2 cells. The cells that penetrated the filter membrane were stained with eosin and counted under the light microscope (magnification $4 \times 10 \times 25$, Fig. 4A). The number of cells penetrating the filter membrane in the shRNA486 group was $14.13 \pm 6.24$, compared with the numbers in the control and negative control groups, which were 29.20 \pm 3.70 and $27.73 \pm 4.47$ cells, respectively (Fig. 4B). No significant difference was observed between the number of cells penetrating the filter membrane in the two control groups $(\mathrm{P}=0.728)$. There was a significant reduction in the number of cells penetrating the filter membrane in the shRNA486 group compared with the two control groups $(\mathrm{P}=0.010$ vs. the control group and $\mathrm{P}=0.015$ vs. the negative control group).

TDRG1 silencing induces the apoptosis of NTERA-2 cells. At 3 days post-transfection, the proportion of apoptotic cells in each group was detected using flow cytometry (Fig. 5). The proportions of early apoptotic cells present in each 
group were as follows: shRNA486, 4.52 $\pm 0.87 \%$; control, $2.49 \pm 0.54 \%$; and negative control, $2.58 \pm 0.88 \%$. The proportions of total apoptotic cells present in each group were as follows: shRNA486, 9.72 $\pm 1.37 \%$; control, $5.97 \pm 0.96 \%$; and negative control, $6.28 \pm 1.26 \%$. The proportions of early apoptotic cells and total apoptotic cells were significantly increased in the shRNA486 group compared with the other two groups $(\mathrm{P}=0.019$ vs. the negative control group and $\mathrm{P}=0.009$ vs. the control group). No significant difference was demonstrated between the control and negative control groups $(\mathrm{P}>0.05)$.

\section{Discussion}

The majority of cases of testiculoma occur in young adults and the incidence is increasing; thus it is important to study its pathogenesis (13). The histological characteristics of testiculomas are complex. Germ cell tumors account for $\sim 95 \%$ of cases, primarily consisting of seminoma and non-seminoma (4). Seminomas account for $~ 50 \%$ of TGCT cases and closely resemble intratubular germ cell neoplasia of an unspecified type (ITGCNU), which is the first step in the development of all TGCTs. Therefore, human seminoma cell lines may be the best cellular models for in vitro studies (14). However, with the exception of primary tumor samples, few tools are available to study the molecular pathogenesis of seminoma (15). To the best of our knowledge and according to the literature, only 3 cell lines (TCam-2, JKT-1 and SEM-1) originate from seminoma (16-18). Furthermore, the origin of these cell lines is not certain; therefore it remains controversial to describe these cell lines as seminoma cell lines $(19,20)$. Taking these reasons into account, the present study used the recognized embryonal carcinoma NTERA-2 cell line as a cell model of TGCT $(21,22)$.

Due to the poor differentiation characteristics and the multiple differentiation potential of ITGCNU, which is the common precursor of the majority of TGCT (23), the composition of TGCT is more closely associated with the choice of therapy compared with other urological neoplasms. Therefore, to determine the causes of differentiation in different types of tumor, studies at the gene and protein levels are particularly important. The onset and development of TGCT are complex processes involving multiple factors. Numerous proto-oncogenes and anti-oncogenes, including p53 and $c$-kit; multiple cell apoptosis genes, including Fas/Fas-L; the expression of the telomerase RNA component; and gene polymorphisms are all implicated in the process of TGCT development (24-28).

TDRG1 is a novel gene that was identified in a previous study by our research group through screening and cloning with digital differential display (7). Reverse transcription-polymerase chain reaction of multiple tissues demonstrated that TDRG1 was expressed in human testes but not in other tissues, such as the heart, liver, brain, epididymis, lung, kidney and spleen. Immunohistochemical staining using a mouse anti-human TDRG1 monoclonal antibody (12) demonstrated that TDRG1 was expressed solely in the testicular seminiferous tubules and spermatogenic cells. No expression was observed in the basal membrane or spermoblasts and a relatively higher expression level was observed in the testes of men between the ages of 15 and 34 years (7). Following analysis of TDRG1 protein expression levels under pathological states, including testiculoma, tuberculocele and testicular atrophy, using tissue microarray technology, another previous study demonstrated that the expression level of TDRGl in the tissues of TGCT, such as seminoma and teratoma, was significantly different compared with non-malignant human testis (8). Furthermore, significant differences were not observed in TDRG1 expression levels between the tuberculocele and testicular atrophy groups (8). The characteristics of TDRG1 expression levels in age, anatomical localization and pathological patterns indicated that TDRG1 may be associated with the oncogenesis, progression or transformation of TGCT.

RNAi degrades target mRNA by introducing exogenous or endogenous double stranded RNA into cells, resulting in inhibition of the corresponding gene (29). In studying the function of a target gene via upregulating or downregulating its expression, it is important to minimize alterations to the functions of other genes through off-target effects. shRNA-mediated RNAi technology is a targeted method for altering the expression of a specific gene (30). In our previously published work, four TDRGl-targeting shRNA recombinant plasmid vectors were successfully constructed using the pGPu6/GFP/Neo (10). psh486 was selected for use in the current study, as it was previously demonstrated the most successful at blocking the expression of the TDRG1 gene. This recombinant vector was successfully transfected into NTERA-2 cells in the present study, and reduced the expression of TDRG1 mRNA in the cells by $79 \%$ at 3 days post-transfection. The expression levels of the TDRG1 protein were also significantly reduced. Therefore, the TDRG1 gene was silenced by psh486 in NTERA-2 cells with high efficiency. NTERA-2 cells display various biological characteristics of tumor cells. At 3 and 5 days following TDRG1 gene silencing by psh486, the in vitro proliferation ability of NTERA-2 cells was inhibited by 15.4 and $26.1 \%$, respectively. Furthermore, at 3 and 5 days, the early apoptotic potential and total apoptotic potential increased by 75 and $54.8 \%$. In addition, the invasion process of NTERA-2 cells was simulated in vitro and demonstrated that the invasive ability of NTERA-2 cells was inhibited by $49.1 \%$ in cells transfected with psh486 compared with untransfected cells.

In conclusion, the present study indicated that the biological behavior of NTERA-2 cells may be closely associated with TDRG1, as the gene may promote the growth and invasion ability of these cells. The gene may be functional in TGCT and can be investigated further as a potential candidate gene in the development of TGCT. However the specific underlying molecular mechanism remains unclear and may involve interactions with other genes. Future studies are required to investigate these remaining questions in addition to the function of TDRG1 in cell lines derived from other testicular tumors.

\section{Acknowledgements}

The present study was supported by grant no. 81372181 from the National Natural Science Foundation of China (www.nsfc.gov.cn). 


\section{References}

1. Horwich A, Shipley J and Huddart R: Testicular germ-cell cancer. Lancet 367: 754-765, 2006.

2. Chia VM, Quraishi SM, Devesa SS, Purdue MP, Cook MB and McGlynn KA: International trends in the incidence of testicular cancer, 1973-2002. Cancer Epidemiol Biomarkers Prev 19: $1151-1159,2010$.

3. Huyghe E, Matsuda T and Thonneau P: Increasing incidence of testicular cancer worldwide: a review. J Urol 170: 5-11, 2003.

4. Winter $\mathrm{C}$ and Albers P: Testicular germ cell tumors: pathogenesis, diagnosis and treatment. Nat Rev Endocrinol 7: 43-53, 2011.

5. Albers P, Albrecht W, Algaba F, et al; European Association of Urology: EAU guidelines on testicular cancer: 2011 update. Eur Urol 60: 304-319, 2011.

6. McIntyre A, Gilbert D, Goddard N, Looijenga L and Shipley J: Genes, chromosomes and the development of testicular germ cell tumors of adolescents and adults. Genes Chromosomes Cancer 47: 547-557, 2008.

7. Jiang X, Li D, Yang J, et al: Characterization of a novel human testis-specific gene: testis developmental related gene 1 (TDRG1). Tohoku J Exp Med 225: 311-318, 2011.

8. Chen HY, Wen JM, Xiao XW, et al: Expression of human testis development related gene 1 in testicular cancer detected by tissue microarray. Zhonghua Nan Ke Xue 16: 883-886, 2010 (In Chinese)

9. Lambeth LS and Smith CA: Short hairpin RNA-mediated gene silencing. Methods Mol Biol 942: 205-232, 2013.

10. Peng S, Yang J, Chen H, et al: Construction of TDRG1 shRNA expression vector and interfering effect of TDRG1 shRNA expression vector on NTERA-2 cells. Zhong Nan Da Xue Xue Bao Yi Xue Ban 37: 979-982, 2012 (In Chinese).

11. Livak KJ and Schmittgen TD: Analysis of relative gene expression data using real-time quantitative PCR and the 2(-Delta Delta C(T)) Method. Methods 25: 402-408, 2001

12. Wen J, Jiang X, Tang Y, Yang J, Chen H and Liu Z: Preparation and identification of monoclonal antibody against human testis development related gene 1. Zhong Nan Da Xue Xue Bao Yi Xue Ban 35: 230-235, 2010 (In Chinese).

13. Rosen A, Jayram G, Drazer M and Eggener SE: Global trends in testicular cancer incidence and mortality. Eur Urol 60: 374-379, 2011.

14. Emerson RE and Ulbright TM: Intratubular germ cell neoplasia of the testis and its associated cancers: the use of novel biomarkers. Pathology 42: 344-355, 2010.

15. Olie RA, Boersma AW, Dekker MC, Nooter K, Looijenga LH and Oosterhuis JW: Apoptosis of human seminoma cells upon disruption of their microenvironment. Br J Cancer 73: 1031-1036, 1996.

16. Kinugawa K, Hyodo F, Matsuki T, et al: Establishment and characterization of a new human testicular seminoma cell line, JKT-1. Int J Urol 5: 282-287, 1998.
17. Mizuno Y, Gotoh A, Kamidono S and Kitazawa S: Establishment and characterization of a new human testicular germ cell tumor cell line (TCam-2). Nihon Hinyokika Gakkai Zasshi 84: 1211-1218, 1993 (In Japanese).

18. Russell SM, Lechner MG, Mokashi A, et al: Establishment and characterization of a new human extragonadal germ cell line, SEM-1, and its comparison with TCam-2 and JKT-1. Urology 81: 464, 2013.

19. de Jong J, Stoop H, Gillis AJ, et al: JKT-1 is not a human seminoma cell line. Int J Androl 30: 350-365, 2007.

20. Eckert D, Nettersheim D, Heukamp LC, Kitazawa S, Biermann K and Schorle H: TCam-2 but not JKT-1 cells resemble seminoma in cell culture. Cell Tissue Res 331: 529-538, 2008

21. Andrews PW, Damjanov I, Simon D, et al: Pluripotent embryonal carcinoma clones derived from the human teratocarcinoma cell line Tera-2. Differentiation in vivo and in vitro. Lab Invest 50: 147-162, 1984.

22. Hasibeder A, Venkataramani V, Thelen P, Radzun HJ and Schweyer S: Phytoestrogens regulate the proliferation and expression of stem cell factors in cell lines of malignant testicular germ cell tumors. Int J Oncol 43: 1385-1394, 2013.

23. Di Vizio D, Cito L, Boccia A, et al: Loss of the tumor suppressor gene PTEN marks the transition from intratubular germ cell neoplasias (ITGCN) to invasive germ cell tumors. Oncogene 24: 1882-1894, 2005.

24. Gutekunst M, Oren M, Weilbacher A, et al: p53 hypersensitivity is the predominant mechanism of the unique responsiveness of testicular germ cell tumor (TGCT) cells to cisplatin. PLoS One 6: e19198, 2011.

25. Biermann K, Göke F, Nettersheim D, et al: c-KIT is frequently mutated in bilateral germ cell tumours and down-regulated during progression from intratubular germ cell neoplasia to seminoma. J Pathol 213: 311-318, 2007.

26. Baldini E, Ulisse S, Marchioni E, et al: Expression of Fas and Fas ligand in human testicular germ cell tumours. Int J Androl 32: 123-130, 2009.

27. Orlando C, Gelmini S, Selli C and Pazzagli M: Telomerase in urological malignancy. J Urol 166: 666-673, 2001.

28. Ruark E, Seal S, McDonald H, et al; UK Testicular Cancer Collaboration (UKTCC): Identification of nine new susceptibility loci for testicular cancer, including variants near DAZL and PRDM14. Nat Genet 45: 686-689, 2013.

29. Fire A, Xu S, Montgomery MK, Kostas SA, Driver SE and Mello CC: Potent and specific genetic interference by double-stranded RNA in Caenorhabditis elegans. Nature 391: 806-811, 1998

30. Moore CB, Guthrie EH, Huang MT and Taxman DJ: Short hairpin RNA (shRNA): design, delivery, and assessment of gene knockdown. Methods Mol Biol 629: 141-158, 2010. 